\title{
A novel 3-hydroxy-3-methylglutaryl-coenzyme A reductase (HMGCR) splice variant with an alternative exon 1 potentially encoding an extended $\mathrm{N}$-terminus
}

\author{
Camilla Stormo $^{1 *}$, Marianne K Kringen ${ }^{2}$, Runa M Grimholt ${ }^{1}$, Jens P Berg ${ }^{1,3}$ and Armin P Piehler ${ }^{1,4}$
}

\begin{abstract}
Background: The major rate-limiting enzyme for de novo cholesterol synthesis is 3-hydroxy-3-methylglutarylcoenzyme A reductase (HMGCR). HMGCR is sterically inhibited by statins, the most commonly prescribed drugs for the prevention of cardiovascular events. Alternative splicing of HMGCR has been implicated in the control of cholesterol homeostasis. The aim of this study was to identify novel alternatively spliced variants of HMGCR with potential physiological importance.

Results: Bioinformatic analyses predicted three novel $H M G C R$ transcripts containing an alternative exon 1 (HMGCR-1b, -1C, -1d) compared with the canonical transcript (HMGCR-1a). The open reading frame of the HMGCR-1b transcript potentially encodes 20 additional amino acids at the N-terminus, compared with HMGCR-1a. Reverse transcription quantitative polymerase chain reaction (RT-qPCR) was used to examine the mRNA levels of HMGCR in different tissues; HMGCR-1a was the most highly expressed variant in most tissues, with the exception of the skin, esophagus, and uterine cervix, in which HMGCR-1b was the most highly expressed transcript. Atorvastatin treatment of HepG2 cells resulted in increased HMGCR-1b mRNA levels, but unaltered proximal promoter activity compared to untreated cells. In contrast, HMGCR-1c showed a more restricted transcription pattern, but was also induced by atorvastatin treatment.
\end{abstract}

Conclusions: The gene encoding HMGCR uses alternative, mutually exclusive exon 1 sequences. This contributes to an increased complexity of HMGCR transcripts. Further studies are needed to investigate whether HMGCR splice variants identified in this study are physiologically functional.

Keywords: 3-hydroxy-3-methylglutaryl-coenzyme A reductase, HMG-CoA, Transcription, Alternative splicing, Statin, Cholesterol

\section{Background}

Human 3-hydroxy-3-methylglutaryl-coenzyme A (HMG$\mathrm{CoA}$ ) reductase (HMGCR) is the rate-limiting enzyme in the isoprenoid/cholesterol biosynthesis pathway. The enzyme converts HMG-CoA to mevalonate and is the main target of commonly used lipid-lowering drugs (i.e., statins) [1]. Some of the major intermediates in the pathway are used to synthesise ubiquinone, dolichol, heme A and isoprenoid lipids [2]. The end-product, cholesterol,

\footnotetext{
* Correspondence: camilla.stormo@medisin.uio.no

1 Department of Medical Biochemistry, University of Oslo and Oslo University Hospital, Ullevål, P.O box 4956, Nydalen, Oslo 0424, Norway

Full list of author information is available at the end of the article
}

is the precursor for the synthesis of crucial compounds, such as steroid hormones, bile acid and vitamin D.

HMGCR is encoded by a single gene on chromosome 5 (5q13.3-q14). Two transcript variants are annotated in the National Center for Biotechnology Information (NCBI) Reference Sequence (RefSeq) database; the canonical full-length transcript composed of 20 exons (NM_000859.2) and an alternatively spliced variant lacking exon 13 (NM_001130996.1). Additionally, exon junction microarray experiments suggest the presence of at least three more $H M G C R$ splice variants lacking exon 8 (AY429542), exon 18 (AY429543) and exons 17 and 18 (AY429544) [3]. 
Expression of HMGCR is strictly regulated at the transcriptional and post-transcriptional level. Cholesterol homeostasis is maintained by $H M G C R$ transcriptional inhibition and enzyme modifications via negative feedback mechanisms (reviewed in [4]). Under sterol-depleted conditions, such as statin treatment, HMGCR transcription is co-ordinately activated with transcription of the low density lipoprotein receptor and other genes in the cholesterol biosynthetic pathway [5].

Genomic diversity is greatly expanded by the process of alternative splicing, by which introns are removed and exons are ligated together to form various mature mRNA transcripts. Alternative splicing has been suggested as another mechanism underlying $\mathrm{HMGCR}$ regulation and cholesterol homeostasis [6]. An HMGCR splice variant lacking exon 13 (denoted here as HMGCR $\Delta$ exon13) was reported to have decreased enzymatic activity and to be less sensitive to statin inhibition $[3,7,8]$. Exon 13 encodes parts of the catalytic domain and the statin binding site of HMGCR $[9,10]$. Recently, the major $A$ allele of a single-nucleotide polymorphism (SNP, rs3846662, $A>G$ ) located within intron 13 of $H M G C R$ was found to be associated with skipping of exon 13 [7]. The relative abundance of HMGCR $\triangle$ exon13 mRNA has also been reported to be associated with interindividual variation in statin-mediated lipid-lowering responses [8,11]. In the present study, nucleotide sequence database queries identified several novel HMGCR splice variants. One of these, HMGCR-1b, shows alternative exon 1 usage, with a novel HMGCR N-terminus predicted. We report the mRNA levels of novel HMGCR splice variants in a variety of healthy human tissues. Furthermore, we present the regulatory responses of these variants in HepG2 cells upon statin treatment.

\section{Methods}

\section{Bioinformatic analysis}

Inspection of displayed mRNA, expressed sequence tag (EST) and RNA sequencing (RNA-seq) tracks at the HMGCR gene locus was performed using the Genome Browser Database provided by the University of California, Santa Cruz (UCSC) on human genome assembly GRCh37/ hg19 [12]. The other bioinformatic tools used were Primer3 software [13], Finch TV1.4.0 (Geospiza, Seattle, USA), DNASTAR Lasergene 7.2 (Madison, WI, USA) and the NCBI database of SNPs.

\section{Cell culture and statin treatment}

The HepG2 cell line (American Type Culture Collection, Manassas, VA, USA) was grown in modified Eagle's minimal essential medium (MEM; ATCC) supplemented with $10 \%$ heat inactivated fetal bovine serum (FBS) and and $1 \times$ penicillin-streptomycin-glutamine (P/S/G) mixture (Sigma-Aldrich, St Louis, MO, USA) in collagen
I-coated tissue culture flasks (BD Biosciences, San Jose, CA, USA). Cells were seeded at $2 \times 10^{5}$ cells $/ \mathrm{mL}$ in 12 well collagen I-coated plates (BD Biosciences), and the statin treatment was carried out in triplicate on the following day. HepG2 cells were cultivated for $24 \mathrm{hrs}$ in MEM with $1 \times \mathrm{P} / \mathrm{S} / \mathrm{G}$ containing $3 \mathrm{mg} / \mathrm{mL}$ of human lipoprotein deficient serum (LPDS; Millipore, Billerica, MA, USA), with or without $10 \mu \mathrm{M}$ of (3 S, 5 S)-Atorvastatin Sodium Salt (Toronto Research Chemicals North York, Ontario, Canada) dissolved in water in a total volume of $1 \mathrm{~mL}$. Two lymphoblastoid cell lines (GM17752 and GM20518; Coriell Institute for Medical Research, Camden, NJ, USA) were cultured according to the manufacturer's instructions. Sequencing confirmed the $C C$ and $A A$ genotypes of the rs3761740 polymorphism in GM17752 and GM20518 cell lines, respectively. Statin treatment was carried out as described above, except that $5 \times 10^{5}$ cells $/ \mathrm{mL}$ of each cell line were seeded into 24-well plates and treated the same day. Cell cultures were maintained at $37^{\circ} \mathrm{C} / 5 \% \mathrm{CO}_{2}$.

\section{Purification of total RNA from cells}

Total RNA from HepG2 cells was isolated using an RNeasy Mini Kit (Qiagen, Venlo, The Netherlands) by following the protocol provided by the manufacturer. DNase digestion with an RNase-Free DNase Set (Qiagen) was used to ensure DNA-free RNA samples. The RNA was eluted in $30 \mu \mathrm{L}$ of RNase/DNase-free water and stored at $-80^{\circ} \mathrm{C}$ until required. RNA samples were quantified using a NanoDrop ND-1000 spectrophotometer (NanoDrop Technologies, Wilmington, DE, USA). RNA quality was assessed by microfluidic capillary electrophoresis using an Agilent 2100 Bioanalyzer and the RNA 6000 Nano Chip kit (Agilent Technologies, Santa Clara, CA, USA). RNA samples were denatured for $2 \mathrm{~min}$ at $70^{\circ} \mathrm{C}$ prior to cDNA synthesis.

\section{Total RNA from human tissues}

High-quality pooled RNA samples from the Human Total Master panel II and the Human Adult Normal Tissue panel were purchased from Clontech (Mountain View, CA, USA) and BioCat GmbH (Heidelberg, Germany), respectively. Ten random RNA samples were assessed for concentration and integrity as described above. All samples were from anonymous donors and commercially available.

\section{Synthesis of cDNA}

The levels of mRNA expression were analysed using total RNA from tissues (1000 ng) and cells (200 ng) in a $20 \mu \mathrm{L}$ cDNA synthesis reaction using an Omniscript RT Kit (Qiagen) and a mixture of $2.5 \mu \mathrm{M}$ oligo-dT and $6.25 \mu \mathrm{M}$ random hexamers (Applied Biosystems, Foster City, CA, USA). Reverse transcription (RT) was performed 
at $37^{\circ} \mathrm{C}$ for $60 \mathrm{~min}$, followed by $95^{\circ} \mathrm{C}$ for $5 \mathrm{~min}$. The cDNA samples were diluted 1:5 in RNase/DNase-free water and stored at $-20^{\circ} \mathrm{C}$ prior to use as templates for PCR amplification. A pooled cDNA sample was obtained by mixing an aliquot of each of the 44 undiluted cDNA samples and a 1:10 dilution in RNase/DNase-free water.

\section{PCR}

Polymerase chain reaction (PCR) amplification of the different first exons of $H M G C R$ in a CDNA pool derived from 44 human tissues (as described above) was performed with HotStarTaq DNA Polymerase (Qiagen) following the manufacturer's instructions Forward primers (exon1a, 5'-GAG CGT GCG TAA GGT GAG G-3'; exon1b, 5'-GAG AGC AGA AGG AAC GCA CA-3'; exon1c, 5'-GAC ATG GTC CTG CAG AGT CG-3'; and exon1d, 5'-GCA GCA TTG CAT AAA TAC TGT CA-3') were used in combination with a reverse primer

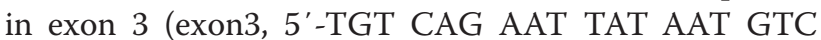
ACT GCT CAA-3'). The cycling conditions were $15 \mathrm{~min}$ at $95^{\circ} \mathrm{C}$, followed by 37 cycles of $30 \mathrm{~s}$ at $94^{\circ} \mathrm{C}, 30 \mathrm{~s}$ at $55^{\circ} \mathrm{C}, 1 \mathrm{~min}$ at $72^{\circ} \mathrm{C}$ and a final extension step at $72^{\circ} \mathrm{C}$ for $10 \mathrm{~min}$. PCR products were separated on $1-3 \%(\mathrm{w} / \mathrm{v})$ agarose gels. Images were captured using a Kodak Image Station 440CF and visualised using Kodak Molecular Imaging Software, version 4.5 (Eastman Kodak Co., New Haven, CT, USA). The PCR products of interest were excised and purified using the QIAquick Gel Extraction Kit (Qiagen) and confirmed by sequencing.

\section{Molecular cloning}

The full-length HMGCR-1b transcript was PCR amplified using a ProofStart DNA polymerase kit (Qiagen) and a cDNA template synthesised from skin (BioCat $\mathrm{GmbH})$. The PCR mix contained $1 \times$ proof start buffer, 1× Q-solution, $0.25 \mathrm{U}$ ProofStart, $5 \mathrm{U}$ HotStarTaq plus DNA polymerase, $350 \mathrm{mM}$ dNTP mix, $2 \mu \mathrm{L}$ of cDNA and $0.4 \mu \mathrm{M}$ forward primer (exon1b, 5'-CGC AGG AGA GGC ACA TTT CAG-3') and reverse primer (exon20, 5'-GGG CTG TCT TCT TGG TGC AAG C-3') in a total volume of $50 \mu \mathrm{L}$. The cycling conditions were $5 \mathrm{~min}$ at $95^{\circ} \mathrm{C}$, followed by 37 cycles of $30 \mathrm{~s}$ at $94^{\circ} \mathrm{C}, 1 \mathrm{~min}$ at $55^{\circ} \mathrm{C}, 3 \mathrm{~min}$ at $72^{\circ} \mathrm{C}$ and a final extension step at $72^{\circ} \mathrm{C}$ for $30 \mathrm{~min}$. Purified PCR products were cloned using the CT-GFP Fusion TOPO TA Expression Kit (Invitrogen Corporation, Carlsbad, CA, USA) according to the manufacturer's instructions. Positive clones were confirmed by sequencing.

\section{Quantitative PCR (qPCR)}

All qPCRs were performed on an ABI 7900HT RealTime PCR System in a standard 96-well format. TaqMan hydrolysis probes (Custom TaqMan Gene Expression Assay from Applied Biosystems) were designed for specific gene quantification of HMGCR-1a, HMGCR-1b and $H M G C R-1 c$. The following TaqMan primer and probe sets were used: 5'-CCA TGC ATT CGA AAA AGT CTT GAC A-3' (forward primer), 5'-GGT TCG GTG GCC TCT AGT G-3' (reverse primer) and the FAMreporter probe $5^{\prime}$-TCC TTG GAT CCT CCA GAT CT-3' for targeting the HMGCR exon 1a-exon 2 boundary; 5'-AAT GGA TGT CGC ACA CAA GAG A-3' (forward primer), 5'-CCA TGC ATT CGA AAA AGT CTT GAC A-3' (reverse primer), and the FAM-reporter probe 5'-CTT GGA TCC TGC GTC TCT-3' for targeting the HMGCR exon 1b-exon 2 boundary; and 5' -TTT CTG GGC TAT ACT AAA TGT GCA TGA-3' (forward primer), 5' -CCA TGC ATT CGA AAA AGT CTT GAC A-3' (reverse primer) and the FAM-reporter probe 5' -ACA AGG ATC CAA GGA TTC-3' for targeting the $H M G C R$ exon 1c-exon 2 boundary. Each qPCR mix comprised $10 \mu \mathrm{L}$ of TaqMan Universal PCR Master Mix, $7 \mu \mathrm{L}$ of RNase-free water, $1 \mu \mathrm{L}$ of TaqMan hydrolysis probe and $2 \mu \mathrm{L}$ of cDNA. The cycling steps were $10 \mathrm{~min}$ at $95^{\circ} \mathrm{C}$ followed by 40 cycles of $95^{\circ} \mathrm{C}$ for $15 \mathrm{~s}$ and $60^{\circ} \mathrm{C}$ for $1 \mathrm{~min}$. Non-template controls were included in each run. All qPCR experiments were performed at least in duplicate. Samples exhibiting more than a 0.5 quantification cycle $(\mathrm{Cq})$ difference between the duplicates were excluded from the relative quantification analysis. Samples showing $\mathrm{Cq}>35$ or undetermined were set to $\mathrm{Cq}=38$ and were considered not detectable.

\section{Reference gene selection}

The stability of 12 reference genes from Applied Biosystems (see [14] for assay IDs) was checked to confirm the reliability of gene expression results across the tissue samples tested. Five (GAPDH, PGK1, SDHA, CTBP1, GOLGA1) were found by geNorm $[15,16]$ to give the most suitable normalisation factors for quantification of relative expression levels. For the atorvastatin treatment experiments in HepG2 cells, the PPIA, TBP, PGK1, $A C T B, G A P D H, U B C, P P I B$, and $S D H A$ reference genes were tested. Two reference genes, PPIA and TBP, were found by geNorm to be the most stable and therefore were suitable for normalisation. The mean expression value was used for the normalisation, according to the " $\Delta \Delta \mathrm{Cq}$ method" for comparing relative expression results between treatments [17]:

$$
\Delta \Delta \mathrm{Cq}=2^{-(\Delta \mathrm{Cqtreated}-\Delta \mathrm{Cq} \text { non-treated })}
$$

\section{Reporter vector construction}

Different regions of the human $H M G C R$ promoter covering -1626 to +12 (relative to the annotated $H M G C R$ transcription start) were amplified by PCR. Genomic DNA from the Human Variation Panels (NA07055; 
Coriell Institute for Medical Research) heterozygous for the HMGCR promoter SNP $C>A$ (rs3761740) were used as PCR templates. The PCR primers were: $5^{\prime}$-AGC GGT ACC GCC ATT TAC ACT AAT GGG TAA AT-3' (forward primer) and 5'-AGT AAG CTT GAC CAA TAA GAG AGG ATC GTT CG-3' (reverse primer) for construction of the reporter vector $1(-559$ to +12$)$; 5'-AGC GGT ACC AAT GGG TAG GCA TAT CCA AGG-3' (forward primer) and 5'-AGT AAG CTT CTG CGT CTT CTG TGC GTT C-3' (reverse primer) for construction of reporter vectors 2 and 3 (-1009 to -615); and 5'-AGC GGT ACC TTG ACA AGG CTG CTA AGA GAA CA-3' (forward primer) for construction of reporter vectors 4 and 5 (-1626 to -615$)$ in combination with the same reverse primer used for reporter vectors 2 and 3. Restriction sites for KpnI and HindIII are underlined in the forward and reverse primer sequences, respectively. The PCR was performed using a Phusion High-Fidelity PCR kit (Finnzymes Oy, Espoo, Finland) according to the manufacturer's instructions. Cycling conditions were as follows: $30 \mathrm{~s}$ at $98^{\circ} \mathrm{C}$, followed by 35 cycles of $10 \mathrm{~s}$ at $98^{\circ} \mathrm{C}, 30 \mathrm{~s}$ at $63^{\circ} \mathrm{C}, 1 \mathrm{~min}$ at $72^{\circ} \mathrm{C}$ and a final extension step of $10 \mathrm{~min}$ at $72^{\circ} \mathrm{C}$. The PCR-amplified promoter regions were cloned into the KpnI and HindIII restriction sites of the promoterless firefly luciferase vector pGL.4.10 (Promega, Madison, WI, USA).

\section{Transfection and reporter gene assay}

HepG2 cells were seeded on the day prior to transfection at $1.25 \times 10^{5}$ cells $/ \mathrm{mL}$ into a 24-well collagen I-coated plate (BD Biosciences) in $0.5 \mathrm{~mL}$ of culture medium. For each transfection, $485 \mathrm{ng}$ of the firefly luciferase empty control vector (pGL4.10) or a test reporter vector was co-transfected with $15 \mathrm{ng}$ of renilla luciferase internal control vector ( $\mathrm{pGL4.73)}$ to normalise for transfection efficiency. Transfection was carried out in triplicate using 1.25 $\mu \mathrm{L}$ of FuGENE HD (Roche Diagnostics Corporation, Indianapolis, IN, USA) reagent per well. Cells were washed with $1 \mathrm{~mL} 1 \times$ phosphate-buffered saline at $24 \mathrm{~h}$ post-transfection and then treated with $100 \mu \mathrm{L}$ of Passive Lysate Buffer supplied in the Dual-Glo luciferase assay kit (Promega). After gentle rocking (15 min), the lysates were assayed for luciferase activity with the Dual-Glo luciferase assay system according to the manufacturer's instructions. Firefly luciferase and renilla luciferase activities were measured using the Wallac 1420 Victor $^{\mathrm{TM}}$ luminometer in a 96-well Wallac B\&W Isoplate ${ }^{\mathrm{TM}}$ (Perkin Elmer, Turku, Finland). Delay and measurement times were set to $2 \mathrm{~s}$ and $10 \mathrm{~s}$, respectively. The linear range of light detection was determined using a dilution series of QuantiLum Recombinant Luciferase (Promega). Firefly luciferase activity was normalised to renilla luciferase expression. Luciferase (Luc) activity induction was calculated as fold-change according to the following equation:

$$
\begin{aligned}
& \text { Fold }- \text { change } \\
& \begin{array}{l}
=[\text { Mean }(\text { Firefly/Renilla)test reporter vector }] \\
/[\text { Mean }(\text { Firefly/Renilla)emptycontrol vector }]
\end{array}
\end{aligned}
$$

\section{Results}

\section{Identification of HMGCR splice variants}

Bioinformatic analysis and visual inspection of the region between HMGCR exon 2 and its adjacent, 5 '-flanking gene in the UCSC genome browser indicated at least three alternative first exons, each spliced to the constitutive exon 2 (Acc. No: AK296499, DA809145, BX952828, designated exons 1b, 1c and 1d, respectively; Figure 1A). Tracks of published RNA-seq data indicated transcription of exon $1 \mathrm{~b}$, whereas no convincing evidence was found for transcription of exons 1c and 1d. Transcription of exons $1 \mathrm{~b}$ and $1 \mathrm{c}$ was confirmed by RT-PCR (Figure 1B) of the cDNA pool derived from 44 different tissues. Expression of the predicted exon 1d could not be confirmed by RT-PCR in any of the investigated tissues. The open reading frame of the canonical HMGCR transcript (=888 amino acids) is maintained in exon 1c, but sequence analysis of exon $1 \mathrm{~b}$ predicted an additional 20 amino acids at the $\mathrm{N}$-terminus. We cloned and sequenced HMGCR-1b (exon 1b- exon 20) and confirmed that exons $1 \mathrm{a}$ and $1 \mathrm{~b}$ were mutually exclusive. We also found that $H M G C R-1 b$ transcripts were subject to exon 13 skipping (HMGCR-1b 13 ) (Figure 1C). Exon $1 \mathrm{~b}$ is potentially translated in certain primates as indicated by alignment of nucleotide sequences from different vertebrate species (Figure 1D).

\section{Tissue distribution of HMGCR-1a, $-1 b$ and $-1 c$}

The RT-qPCR experiments showed variable levels of alternative first exons of $H M G C R$ in 36 different tissues (Figure 2). The canonical HMGCR-1a transcript was ubiquitously expressed at the highest levels in the cerebellum, fetal brain, testis, skin and adrenal gland (Figure 2A). The highest expression of HMGCR-1b was detected in the skin, esophagus, uterine cervix and prostate (Figure 2B). HMGCR-1b mRNA levels were below detection levels $(\mathrm{Cq}>35.0)$ in six tissues. The level of HMGCR-1a in liver (pooled from three different donors) was approximately two-fold higher than that of $H M G C R-1 b$. We observed higher HMGCR-1a expression in most of the tissues analysed with the exception of the skin, esophagus, uterine cervix and colon, in which the levels of $H M G C R-1 b$ were higher (approximately 1.5-2.0-fold; Figure 2D). HMGCR-1c was detectable $(\mathrm{Cq}<35)$ in only nine of the tissues analysed and was less abundant than HMGCR-1a. The highest expression of 


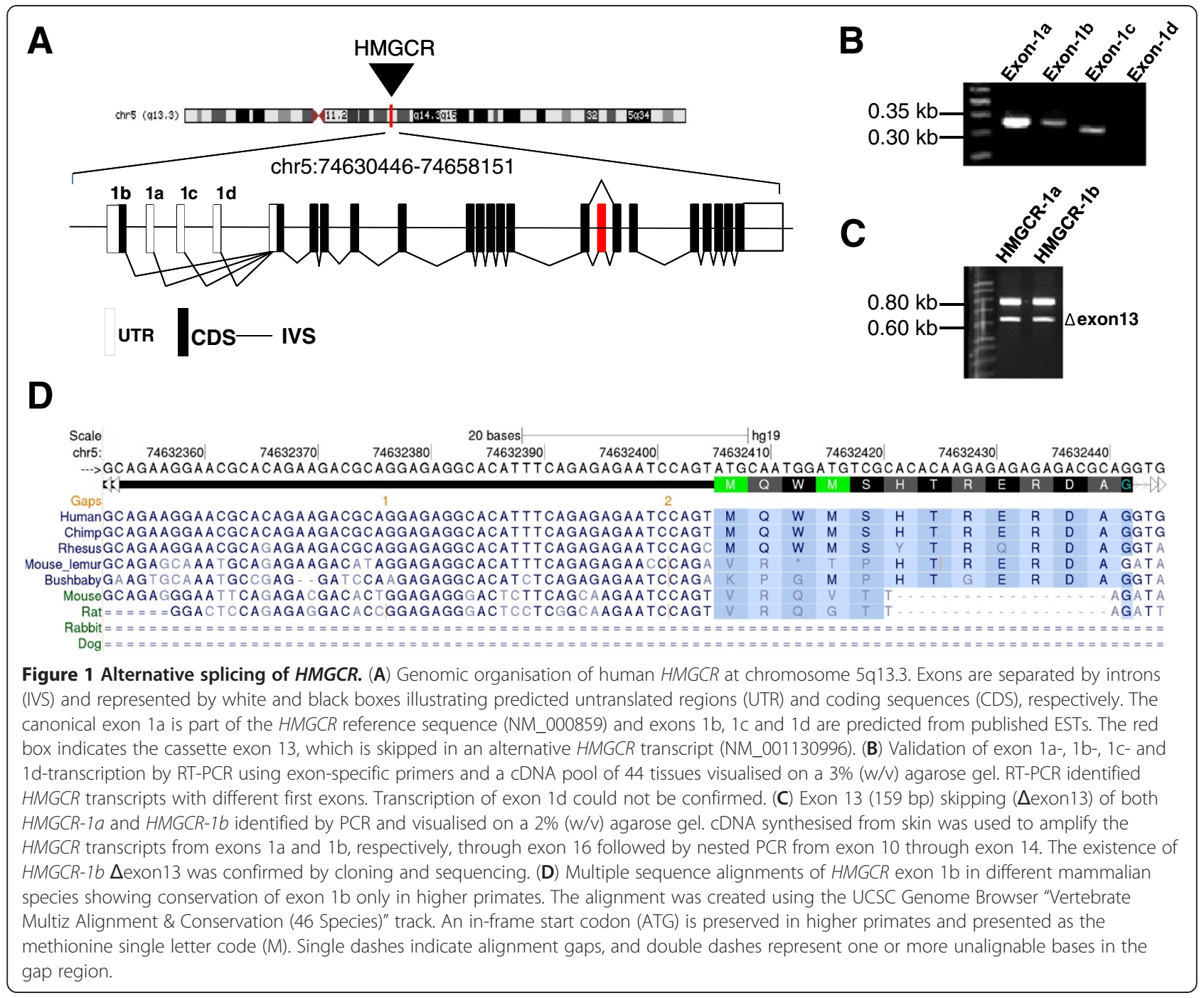

HMGCR-1c was detected in the cerebellum, fetal brain, testis and adrenal gland (Figure 1C). HMGCR-1c could not be quantified in the skin or uterine cervix $(\mathrm{Cq}>35)$, where $H M G C R-1 b$ was relatively abundant.

\section{Expression analysis before and after atorvastatin treatment in HepG2 cells}

HepG2 cells, a model system for cholesterol biosynthesis studies, were treated with atorvastatin for $24 \mathrm{~h}$. The RT-qPCR experiments showed significant mRNA induction $(p<0.05)$ for HMGCR-1a $(2.1 \pm 0.2$-fold (mean $\pm \mathrm{SE}$; Figure 3A), HMGCR-1b (1.6 \pm 0.2 -fold; Figure 3B) and HMGCR-1c (2.6 \pm 0.3 -fold; Figure 3C) in cells after atorvastatin treatment compared with untreated cells. The magnitude of induction was significantly different between HMGCR-1a and HMGCR-1b, and between $H M G C R-1 b$ and HMGCR-1c $(p<0.05)$, but not between HMGCR-1a and HMGCR-1c $(p=0.16)$. The HMGCR-1c and $H M G C R-1 b$ splice variants were less abundant (approximately 60-fold lower) in HepG2 cells compared with the canonical HMGCR-1a. The fact that exon $1 \mathrm{~b}$ was found to be approximately two-fold lower than exon $1 \mathrm{a}$ in the liver suggests that there are major differences in the expression levels of these transcripts, either between different liver donors or because of cell immortalisation and/or an artificial cell culture environment for the HepG2 cells.

\section{HMGCR promoter analysis}

Bioinformatics showed that exon $1 \mathrm{~b}$ is located $35 \mathrm{bp}$ upstream of the CpG island in the previously characterised $H M G C R$ promoter. We performed luciferase reporter gene assays in HepG2 cells under various conditions to investigate the promoter activity driving the transcription of HMGCR-1b and the genetic influence of the promoter SNP rs3761740, which is close to exon 1b. The 


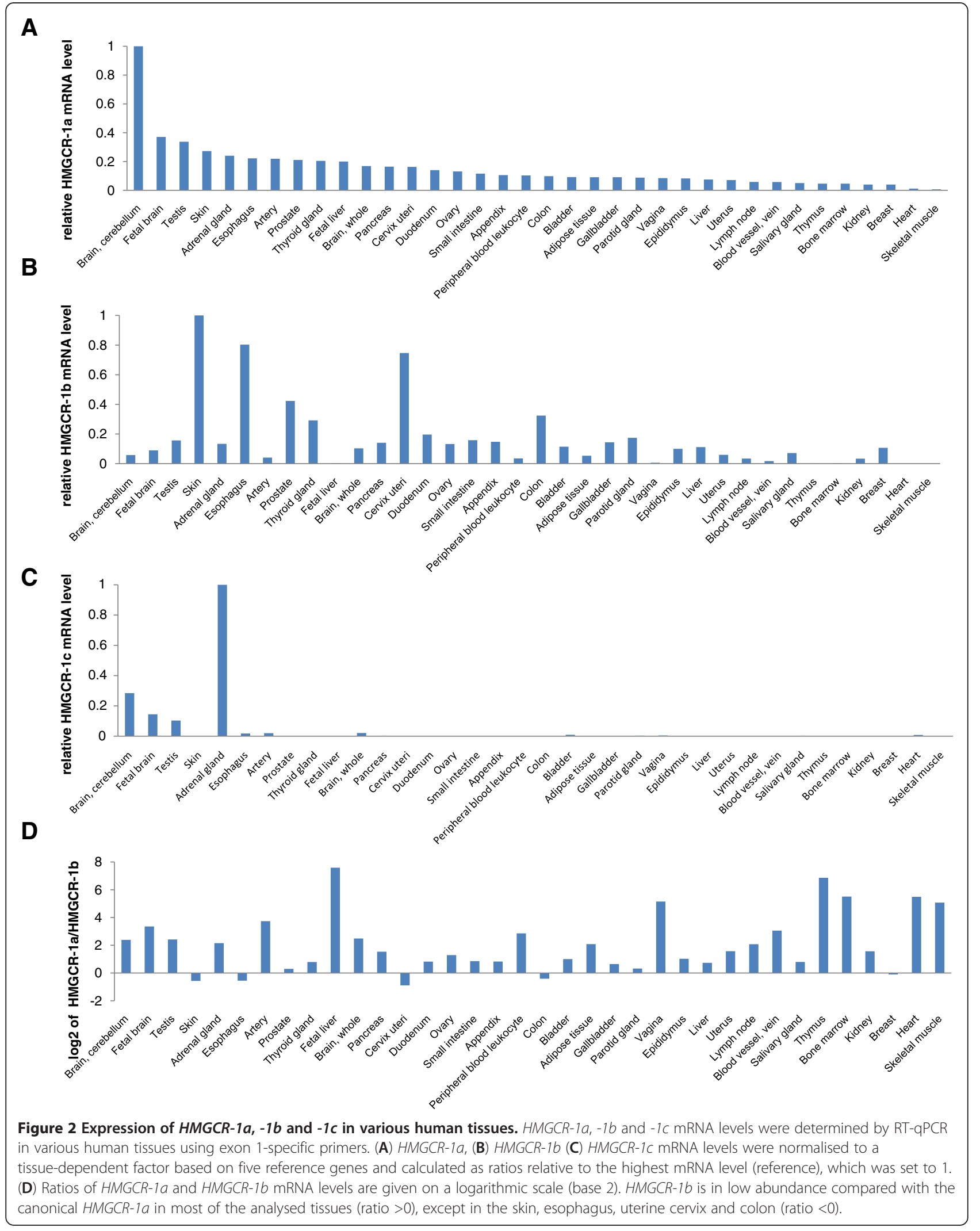



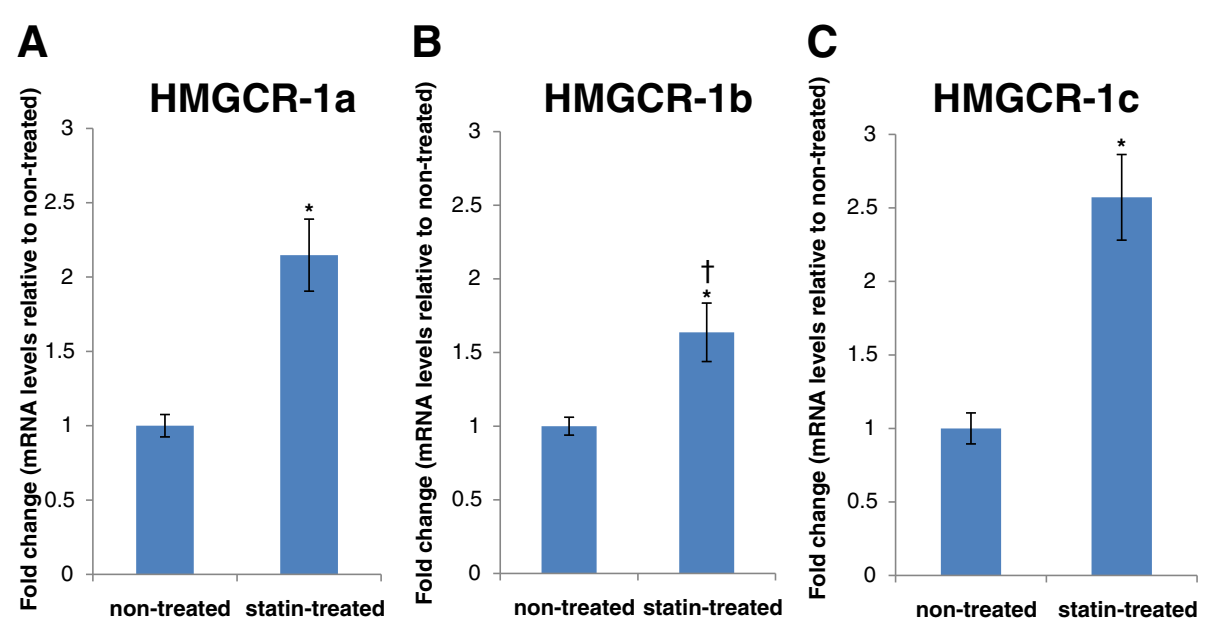

Figure 3 Upregulation of HMGCR transcript variants in HepG2 cells treated with atorvastatin. HepG2 cells were incubated in culture medium with lipoprotein deficient serum (LPDS; non-treated) and $10 \mu \mathrm{M}$ atorvastatin + LPDS for $24 \mathrm{~h}$ (treated). The mRNA levels of (A) HMGCR-1a (B) HMGCR-1b and (C) HMGCR-1c were measured by RT-qPCR using exon1-specific primers and a set of validated reference genes for normalisation. The results are shown as the mean fold-change compared with mRNA levels in non-treated cells. Error bars indicate standard errors ( \pm S.E), $n=5$. *Statistically significant differences between non-treated and treated $(p<0.05)$ were determined using Student's $t$-test. $†$ The magnitude of statin-induced upregulation of HMGCR-1b was significantly lower $(p<0.05)$ than that of HMGCR-1a and HMGCR-1C, respectively, as determined by a paired-sample Student's t-test.

promoter region upstream of exon 1a (reporter vector 1, which was used as a positive control) was active under standard culture conditions with 10\% FBS (Figure 4). The exon 1a promoter was further activated under sterol-depleted conditions with LPDS $(1.7 \pm 0.2$-fold $)$ and LPDS + atorvastatin (3.0 \pm 0.6 -fold) when compared with the activity of transfected cells cultured under standard conditions, containing $10 \%$ FBS. The reporter vectors 2,

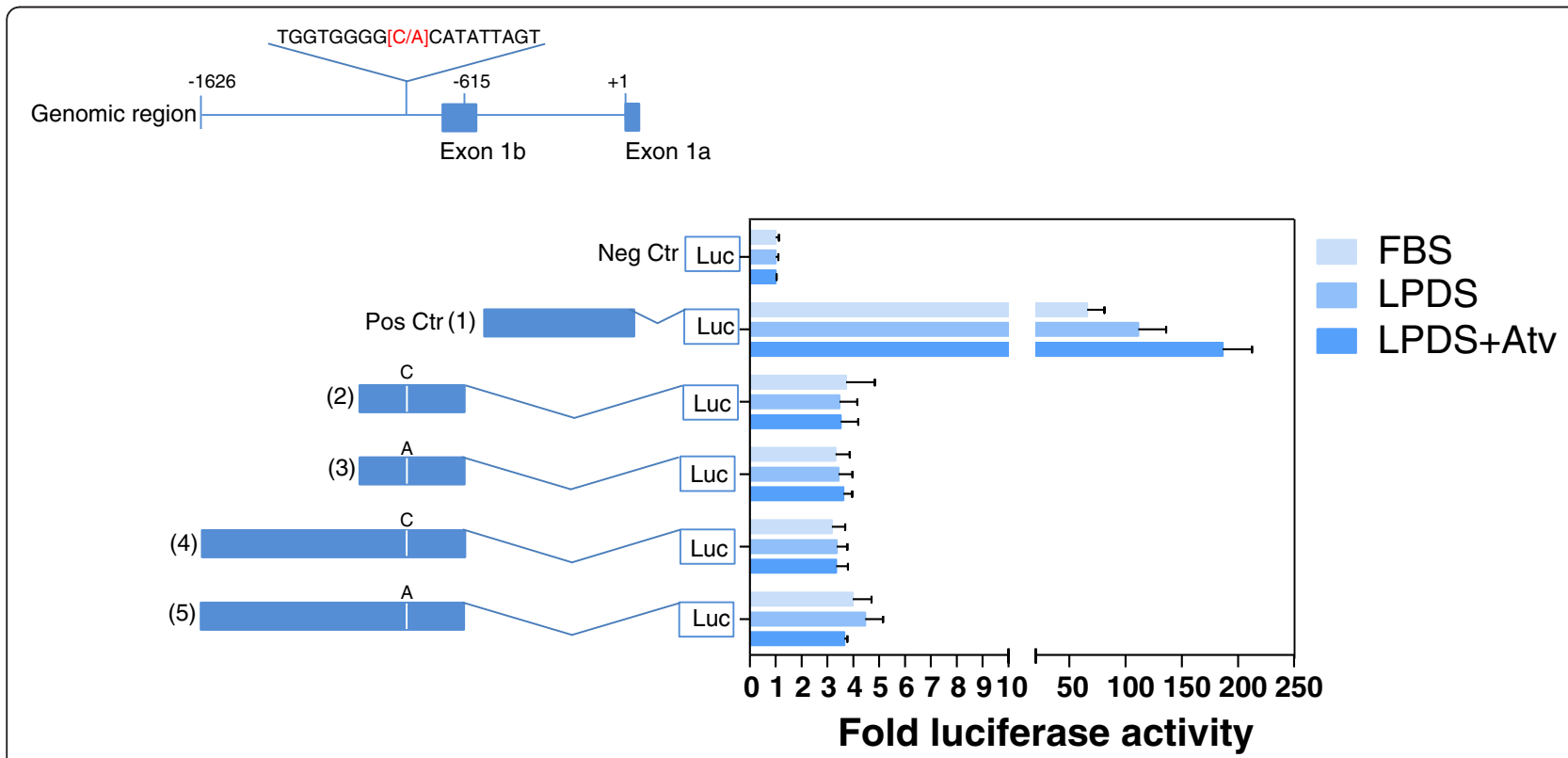

Figure 4 Promoter activities of the canonical HMGCR-1a and the putative HMGCR-1b promoter in HepG2 cells. Solid bars illustrate different sizes of genomic sequences upstream of exon $1 \mathrm{a}$ and exon $1 \mathrm{~b}$ that were cloned into pGL4.10. The HMGCR promoter SNP (C>A, rs3761740) is highlighted in red and represented by both alleles. Luciferase activity was measured in the lysates of transfected cells incubated for $24 \mathrm{~h}$ in fetal bovine serum (FBS), lipoprotein-deficient serum (LPDS) or LPDS with $10 \mu \mathrm{M}$ atorvastatin (Atv). The promoter activity is shown as the mean fold-change in firefly luciferase activity relative to the mean firefly luciferase activity of the empty pGL4.10 vector, which was set to 1 after correction for transfection efficiency using Renilla luciferase activity. The results are the mean of three independent experiments performed in triplicate. Error bars indicate standard errors ( \pm S.E). 
3, 4 and 5, each containing genomic fragments upstream of exon $1 \mathrm{~b}$, exhibited low promoter activities that were not induced by sterol depletion (LPDS), or by higher degrees of sterol depletion (LPDS + atorvastatin). The minor $A$ allele of the promoter SNP $C>A$ (rs3761740) did not affect the promoter activity of exon $1 \mathrm{~b}$ when compared with the major allele $C$ reporter vector. The effect of the promoter SNP $C>A$ (rs3761740) on HMGCR exons $1 \mathrm{a}$ and $1 \mathrm{~b}$ mRNA levels was investigated after atorvastatin treatment in two different lymphoblastoid cell lines homozygous for either allele. The RTqPCR experiments $(n=5)$ showed similar induction of HMGCR-1b mRNA levels after atorvastatin treatment in both lymphoblastoid cell lines, independent of the allelic status. HMGCR-1b was induced 1.2 \pm 0.1 -fold (mean \pm $\mathrm{SE})$ and $1.3 \pm 0.1$-fold in cells with the major $C C$ and the minor $A A$ genotype of $\mathrm{rs} 3761740$, respectively. The magnitude of induction was not significantly different between the two different cell lines. The canonical HMGCR-1a transcript was upregulated $1.7 \pm 0.1$-fold in both lymphoblastoid cell lines after atorvastatin treatment.

\section{Discussion}

HMGCR is the key enzyme in the cholesterol biosynthesis pathway and is the major target of cholesterollowering statin drugs. Here, we demonstrate the existence of alternative exon 1 usage in human $H M G C R$, resulting in several novel transcript variants. One of these, HMGCR-1b, encodes a novel N-terminus which potentially extends the membrane domain by 20 amino acids compared with the canonical sequence. The canonical HMGCR protein is composed of 888 amino acids with a regulatory, sterol-sensing transmembrane domain (residues 10 - 339), a flexible linker (residues 340 - 449) and a C-terminal catalytic region (residues $450-888$ ) [18]. We studied HMGCR-1b at the genomic and transcriptional levels. Whether the translation machinery actually recognises the first AUG as a start codon to produce a protein with 20 additional amino acids in the membrane domain remains to be determined. Unfortunately, initial experiments using rabbit antibodies raised against the novel N-terminus of the predicted HMGCR-1b polypeptide exhibited non-specific binding (data not shown).

In mammalian genomes, only one gene encodes the HMGCR enzyme, which mainly resides in the membrane of the endoplasmic reticulum $[19,20]$. A minor fraction (approximately 5\%) of the reductase activity has been demonstrated in rat liver [21-23] and mouse brain [24] to be localised in the matrix of peroxisomes. The signal that targets HMGCR to peroxisomes has not yet been determined. The HMGCR-1b transcript identified in this study is remarkably less abundant than the canonical HMGCR-1a in most of the tissues analysed, and it is tempting to speculate that exon $1 \mathrm{~b}$ might encode the missing HMGCR peroxisomal targeting information [25]. However, bioinformatic analysis of the $\mathrm{N}$-terminus of the hypothetical HMGCR-1b protein did not reveal any peroxisomal targeting signals (data not shown).

Our finding that $H M G C R$ generates multiple $5^{\prime}$ end transcripts is not unusual in the human genome [26]. There are numerous other examples in the literature. The gene locus encoding the uridine diphosphateglucuronosyltransferase 1 family contains several different coding first exons, which generate at least nine different transcripts encoding various isozymes with unique N-termini and substrate specificities [12]. Another example is the CYP19A1 gene encoding aromatase, the rate-limiting enzyme of estrogen biosynthesis, which contains alternative non-coding first exons affecting RNA stability and aromatase levels in tissues. Differential use of non-coding first exons is suggested to be an important mechanism of post-transcriptional regulation of aromatase gene expression [27].

Whether an alternatively spliced variant is functional is considered in the context of tissue-specific expression and conservation in most studies [28]. The mRNA levels for the alternative $H M G C R-1 b$ splice variant were more abundant than the canonical HMGCR-1a levels in epithelial tissues, such as skin. Skin is one of the major sites for cholesterol production in the body [29], where HMGCR activity and cholesterol synthesis are crucial for hindering fluid loss from the body by maintaining the permeability barrier [30]. In addition, the production of non-cholesterol isoprenoid intermediates by HMGCR has been reported to be important during acute wound healing [31]. Sequence alignment of $H M G C R-1 b$ did not show conservation of the open reading frame in all terrestrial animals. Therefore, it remains to be determined whether the HMGCR-1b splice variant exerts an epithelial or skin-specific role in sustaining the epithelial barrier function in certain primates.

Tissue-specific gene expression may be regulated via differential gene promoter activity [32]. The weak promoter activity of the genomic region upstream of exon $1 \mathrm{~b}$ is in accordance with the rather low, basal mRNA expression level of exon 1b found in HepG2 cells. Unexpectedly, the alternative promoter activity in the reporter assay was not induced by sterol depletion, although there was an increase in the HMGCR-1b mRNA level in HepG2 cells. Based on these results, the increased $H M G C R-1 b$ mRNA level due to sterol depletion in HepG2 cells most likely results from post-transcriptional regulation and/or regulatory promoter DNA sequences not captured in our reporter vector constructs. Moreover, previous studies have identified a functional $C>A$ 
SNP (rs3761740) in the HMGCR promoter [23,33]. Keller et al. identified a sterol regulatory element (SRE) in this position. They found that the minor $A$ allele was more responsive to the overexpression of sterol regulatory element-binding protein 2, compared with the major $C$ allele [33]. In our experiments, this promoter SNP, which is close to the transcription start of exon $1 \mathrm{~b}$, did not contribute significantly to the proximal promoter activity or mRNA level of $H M G C R-1 b$ or to the transcription of the canonical HMGCR-1a variant upon sterol depletion.

Approximately half of human genes are predicted to have alternative promoters [34]. A comparative study of different promoter features in human and mouse genes reported a distinct class of "non-conserved" promoters in humans [35]. Alternative promoters differ from the major promoter in their responsiveness to a specific condition, resulting in different expression levels in tissues or developmental stages and thereby increasing the flexibility of gene expression. Given that the putative $H M G C R-1 b$ promoter identified in our study (i) exhibits a different response to statin treatment compared with the canonical $H M G C R$ promoter, (ii) is of minor usage, (iii) is lacking CpG islands, and (iv) that the HMGCR-1b transcript shows an expression pattern distinct from that of $H M G C R-1 a$, it seems likely that the HMGCR-1b promoter represents an alternative, non-conserved $H M G C R$ promoter that contributes to the expressional diversity of this gene.

With respect to HMGCR-1a, we also found that exon 13 , which encodes amino acids in the catalytic region, is a cassette exon in the $H M G C R-1 b$ transcript, meaning that this exon is either included (HMGCR-1b) or skipped (HMGCR-1bD13). The catalytically active HMGCR is formed as a tetramer with two dimers [10]. Medina et al. have proposed that the inactive HMGCR lacking exon 13 modulates the HMGCR enzyme activity by being part of the catalytically active tetramer and thereby reducing its total enzyme activity [11]. Further studies are needed to clarify whether the delta-exon 13 variant of the $H M G C R-1 b$ transcript also contributes to HMGCR enzyme activity modulation and statin response.

\section{Conclusions}

We have shown that human HMGCR uses different first exons to produce multiple alternatively spliced variants, thereby contributing to increased transcriptional complexity. We have designated these splice variants HMGCR-1a, HMGCR-1b and HMGCR-1c. The novel $H M G C R$ exon 1 (HMGCR-1b) we identified contains an in-frame ATG translational start codon predicting an extended N-terminus of HMGCR. Moreover, we showed that this transcript variant is differentially expressed in a wide variety of healthy human tissues, and that its
mRNA levels are regulated upon statin treatment. Further studies are needed to elucidate potential physiological roles of differential first exon usage in HMGCR.

\section{Abbreviations}

HMGCR: 3-Hydroxy-3-Methylglutaryl-Coenzyme A reductase; HMG-CoA: 3-Hydroxy-3-Methylglutaryl-Coenzyme A; SNP: Single-Nucleotide Polymorphism; EST: Expressed Sequence Tag; RNA-seq: RNA sequencing; MEM: Modified Eagle's Minimal essential medium; FBS: Fetal Bovine Serum; LPDS: Lipoprotein Deficient Serum; RT: Reverse Transcription; PCR: Polymerase Chain Reaction; qPCR: Quantitative Polymerase Chain Reaction; Luc: Luciferase; Atv: Atorvastatin.

\section{Competing interests}

The authors declare that they have no competing interests.

\section{Authors' contributions}

CS carried out the experimental work and drafted the manuscript with assistance from RMG and MKK. AP, MKK and JPB participated in the study design, interpretation of data and revising the manuscript. All authors read and approved the final manuscript.

\section{Acknowledgements}

We give special thanks to Daniel Sachse for technical assistance with figure illustrations and statistical analysis. We thank Dr. Bernd Thiede for help with protein bioinformatics. This work was funded by the Division of Diagnostics and Intervention, Oslo University Hospital, Norway and South-Eastern Norway Regional Health Authority.

\section{Author details}

'Department of Medical Biochemistry, University of Oslo and Oslo University Hospital, Ullevål, P.O box 4956, Nydalen, Oslo 0424, Norway. ${ }^{2}$ Department of Pharmacology, Oslo University Hospital, Ullevål, P.O box 4956, Nydalen, Oslo 0424, Norway. ${ }^{3}$ Institute of Clinical Medicine, Faculty of Medicine, University of Oslo, P.O box 1171, Blindern, Oslo 0318, Norway. ${ }^{4}$ Fürst Medical Laboratory, Søren Bullsvei 25, Oslo NO-1051, Norway.

Received: 3 January 2012 Accepted: 11 July 2012

Published: 18 September 2012

\section{Reference}

1. Maron DJ, Fazio S, Linton MF: Current perspectives on statins. Circulation 2000, 101:207-213.

2. Goldstein JL, Brown MS: Regulation of the mevalonate pathway. Nature 1990, 343:425-430

3. Johnson JM, Castle J, Garrett-Engele P, Kan Z, Loerch PM, Armour CD, Santos R, Schadt EE, Stoughton R, Shoemaker DD: Genome-wide survey of human alternative pre-mRNA splicing with exon junction microarrays. Science 2003, 302:2141-2144.

4. DeBose-Boyd RA: Feedback regulation of cholesterol synthesis: sterolaccelerated ubiquitination and degradation of HMG CoA reductase. Cell Res 2008, 18:609-621.

5. Horton JD, Goldstein JL, Brown MS: SREBPs: activators of the complete program of cholesterol and fatty acid synthesis in the liver. $J$ Clin Invest 2002, 109:1125-1131.

6. Medina MW, Gao F, Naidoo D, Rudel LL, Temel RE, McDaniel AL, Marshall SM, Krauss RM: Coordinately regulated alternative splicing of genes involved in cholesterol biosynthesis and uptake. PLoS One 2011, 6:e19420.

7. Burkhardt R, Kenny EE, Lowe JK, Birkeland A, Josowitz R, Noel M, Salit J, Maller JB, Pe'er I, Daly MJ: Common SNPs in HMGCR in micronesians and whites associated with LDL-cholesterol levels affect alternative splicing of exon13. Arterioscler Thromb Vasc Biol 2078, 2008:28.

8. Medina MW, Gao F, Ruan W, Rotter Jl, Krauss RM: Alternative splicing of 3-hydroxy-3-methylglutaryl coenzyme A reductase is associated with plasma low-density lipoprotein cholesterol response to simvastatin. Circulation 2008, 118:355-362.

9. Istvan ES, Deisenhofer J: Structural mechanism for statin inhibition of HMG-CoA reductase. Science 2001, 292:1160-1164.

10. Istvan ES, Palnitkar M, Buchanan SK, Deisenhofer J: Crystal structure of the catalytic portion of human HMG-CoA reductase: insights into regulation of activity and catalysis. EMBO J 2000, 19:819-830. 
11. Medina MW, Krauss RM: The role of HMGCR alternative splicing in statin efficacy. Trends Cardiovasc Med 2009, 19:173-177.

12. UCSC Genome Browser. http://genome.ucsc.edu/.

13. Rozen S, Skaletsky H: Primer3 on the WWW for general users and for biologist programmers. Methods Mol Biol 2000, 132:365-386.

14. Piehler AP, Grimholt RM, Ovstebo R, Berg JP: Gene expression results in lipopolysaccharide-stimulated monocytes depend significantly on the choice of reference genes. BMC Immunol 2010, 11:21.

15. Vandesompele J, De PK, Pattyn F, Poppe B, Van RN, De PA, Speleman F: Accurate normalization of real-time quantitative RT-PCR data by geometric averaging of multiple internal control genes. Genome Biol 2002, 3:RESEARCH0034.

16. geNorm. http://medgen.ugent.be/ jvdesomp/genorm/.

17. Pfaffl MW: A new mathematical model for relative quantification in realtime RT-PCR. Nucleic Acids Res 2001, 29:e45.

18. Protein knowledgebase (UniProtKB). http://www.uniprot.org/.

19. Engfelt WH, Masuda KR, Paton VG, Krisans SK: Splice donor site mutations in the 3-hydroxy-3-methylglutaryl coenzyme A reductase gene cause a deficiency of the endoplasmic reticulum 3-hydroxy-3-methylglutaryl coenzyme A reductase protein in UT2 cells. J Lipid Res 1998, 39:2182-2191.

20. Liscum L, Finer-Moore J, Stroud RM, Luskey KL, Brown MS, Goldstein JL: Domain structure of 3-hydroxy-3-methylglutaryl coenzyme A reductase, a glycoprotein of the endoplasmic reticulum. J Biol Chem 1985, 260:522-530.

21. Keller GA, Barton MC, Shapiro DJ, Singer SJ: 3-Hydroxy-3-methylglutarylcoenzyme $\mathrm{A}$ reductase is present in peroxisomes in normal rat liver cells. Proc Natl Acad Sci U S A 1985, 82:770-774.

22. Keller GA, Pazirandeh M, Krisans S: 3-Hydroxy-3-methylglutaryl coenzyme A reductase localization in rat liver peroxisomes and microsomes of control and cholestyramine-treated animals: quantitative biochemical and immunoelectron microscopical analyses. J Cell Biol 1986, 103:875-886.

23. Porcellini E, Calabrese E, Guerini F, Govoni M, Chiappelli M, Tumini E, Morgan K, Chappell S, Kalsheker N, Franceschi M, et al: The hydroxymethyl-glutaryl $\mathrm{CoA}$ reductase promoter polymorphism is associated with Alzheimer's risk and cognitive deterioration. Neurosci Lett 2007, 416:66-70.

24. Kovacs WJ, Faust PL, Keller GA, Krisans SK: Purification of brain peroxisomes and localization of 3-hydroxy-3-methylglutaryl coenzyme A reductase. Eur J Biochem 2001, 268:4850-4859.

25. Breitling R, Krisans SK: A second gene for peroxisomal HMG-CoA reductase? A genomic reassessment. J Lipid Res 2002, 43:2031-2036.

26. Davuluri RV, Suzuki Y, Sugano S, Plass C, Huang TH: The functional consequences of alternative promoter use in mammalian genomes. Trends Genet 2008, 24:167-177.

27. Wang H, Li R, Hu Y: The alternative noncoding exons 1 of aromatase (Cyp19) gene modulate gene expression in a posttranscriptional manner. Endocrinology 2009, 150:3301-3307.

28. Melamud E, Moult J: Stochastic noise in splicing machinery. Nucleic Acids Res 2009, 37:4873-4886.

29. Feingold KR, Wiley MH, Moser AH, Lau DT, Lear SR, Siperstein MD: De novo sterologenesis in intact primates. J Lab Clin Med 1982, 100:405-410.

30. Lampe MA, Burlingame AL, Whitney J, Williams ML, Brown BE, Roitman E, Elias PM: Human stratum corneum lipids: characterization and regional variations. J Lipid Res 1983, 24:120-130.

31. Schiefelbein D, Goren I, Fisslthaler B, Schmidt H, Geisslinger G, Pfeilschifter J, Frank S: Biphasic regulation of HMG-CoA reductase expression and activity during wound healing and its functional role in the control of keratinocyte angiogenic and proliferative responses. J Biol Chem 2008, 283:15479-15490.

32. Ayoubi TA, Van De Ven WJ: Regulation of gene expression by alternative promoters. FASEB J 1996, 10:453-460.

33. Keller L, Murphy C, Wang HX, Fratiglioni L, Olin M, Gafvels M, Bjorkhem I, Graff C, Meaney S: A functional polymorphism in the HMGCR promoter affects transcriptional activity but not the risk for Alzheimer disease in Swedish populations. Brain Res 2010, 1344:185-191.

34. Cooper SJ, Trinklein ND, Anton ED, Nguyen L, Myers RM: Comprehensive analysis of transcriptional promoter structure and function in $1 \%$ of the human genome. Genome Res 2006, 16:1-10.

35. Tsuritani K, rrie T, Yamashita R, Sakakibara Y, Wakaguri H, Kanai A, Mizushima-Sugano J, Sugano S, Nakai K, Suzuki Y: Distinct class of putative "non-conserved" promoters in humans: comparative studies of alternative promoters of human and mouse genes. Genome Res 2007, 17:1005-1014

doi:10.1186/1471-2199-13-29

Cite this article as: Stormo et al:: A novel 3-hydroxy-3-methylglutarylcoenzyme A reductase (HMGCR) splice variant with an alternative exon 1 potentially encoding an extended $\mathrm{N}$-terminus. BMC Molecular Biology 2012 13:29.

\section{Submit your next manuscript to BioMed Central and take full advantage of:}

- Convenient online submission

- Thorough peer review

- No space constraints or color figure charges

- Immediate publication on acceptance

- Inclusion in PubMed, CAS, Scopus and Google Scholar

- Research which is freely available for redistribution

Submit your manuscript at www.biomedcentral.com/submit
Biomed Central 